\title{
Computation of changes in explosive weather systems during the 20th century
}

\author{
S. L. Osbrough ${ }^{1} \quad$ J. S. Frederiksen ${ }^{2}$
}

Received 6 February 2017; revised 13 November 2017

\begin{abstract}
We present a new computational technique for extracting growing weather modes of different frequencies and growth rates from instantaneous six hourly observed atmospheric data. The methodology is applied to examine the changes in the statistics of growing storms and extreme weather events between the mid and late 20th century. The structures of weather modes in different frequency and growth rate bands are determined from the leading (maximum variance) eigenvectors of the associated covariance matrices. A matrix stochastic model is also fitted to the data, with the associated leading eigenvectors related to the dynamical developments.
\end{abstract}

DOI:10.21914/anziamj.v58i0.11784, (c) Austral. Mathematical Soc. 2017. Published 2017-11-28, as part of the Proceedings of the 18th Biennial Computational Techniques and Applications Conference. ISSN 1445-8810. (Print two pages per sheet of paper.) Copies of this article must not be made otherwise available on the internet; instead link directly to the DOI for this article. Record comments on this article via http://journal. austms . org.au/ojs/index . php/ANZIAMJ/comment/add/11784/0 


\section{Contents}

1 Introduction

C138

2 Datasets and high pass filtering

C139

3 POPs and EOFs

C140

4 Computation of growing disturbances

C143

5 Conclusion

C145

References

C147

\section{Introduction}

Recent changes in the mean circulation patterns of the southern hemisphere (SH) have had a dramatic effect on storm development and rainfall across parts of Australia $[4,5,7]$. There was a $20 \%$ decrease in winter rainfall in southwest Western Australia (SWWA) since the 1970s. During the peak of the Australian Millennium Drought (AMD), between 1997 and 2006, south-eastern Australia experienced the most persistent rainfall deficit since the start of the 20th century [7]. Primary causes of the drying of SWwA during the AMD were found to be a reduction in the growth rates of leading storm track modes and a deflection of some storms south of the Australian continent $[4,5,6]$. The role of extra tropical cyclogenesis in the rainfall reduction was established by solving large eigenvalue-eigenvector problems for the leading stormtrack modes in a primitive equation instability model [4, 5, 6, 7]. These were solved using both direct and Arnoldi-Krylov subspace reduction methods [5]. As well it was shown that a simple diagnostic, the Phillips Criterion, which measures the vertical wind shear and the static stability (vertical potential temperature shear), could be used to understand broad features of the changes. 
The peak SH jet stream was found to have reduced by nearly $20 \%$ since the 1970s. The instability calculations capture the average change in growth rates of leading modes over given long time periods, but do not give information on extreme events or on the statistics of change in growth rates and the number of associated growing storms. Here we develop a new method for answering these questions by calculating the growing storm track disturbances directly from the 6 hourly National Centers for Ensemble Prediction (NCEP) reanalysed observed dataset of atmospheric flows.

We begin this article by outlining the process by which data is filtered to calculate the growth rates of storm tracks. In Section 3 statistical methods are employed to examine the validity of this filtering process. A new computational technique is then introduced in Section 4 using realistic atmospheric flows from observational reanalysis data to provide statistics about growing disturbances. We apply our method to a case study of changes in storm development statistics during the AMD compared with the earlier 20 year period (19491968) and draw comparisons with previous work mentioned above. Concluding remarks and discussion on possible future studies are presented in Section 5.

\section{Datasets and high pass filtering}

We use the 6 hourly NCEP reanalysis data for determining changes in growing (and decaying) storms during the 20th century. Firstly, the annual cycle is removed by subtracting the average of a ten day running mean over the years of interest from the instantaneous data. Secondly, the data is filtered with a high pass filter that only accepts periods less than 4 days, which are typical of the storms of interest. The high pass filter is determined by: $R_{H}(\omega)=1-R_{L}(\omega)$, where $\omega$ is the angular frequency and the low pass filter, $R_{L}$, is constructed by using a symmetric running mean shaped by the sinc function. Thus, if the input timeseries is $X(t)$, where $t$ is time, the new low 
pass time series $\mathrm{Y}(\mathrm{t})$ is given by Baxter and King [1]

$$
Y(t)=\sum_{k=-K}^{K} C_{k} X(t+k \Delta t),
$$

where $C_{-k}=C_{k}$ with $C_{k}=1 /(k \pi) \sin (k \hat{\omega})$ and $C_{0}=\hat{\omega} / \pi$, where $\hat{\omega}$ is the cutoff angular frequency. For a low pass filter that accepts periods greater than 4 days and with a time step of $\Delta t=6$ hours we have $\hat{\omega}=\pi / 8$ day $^{-1}$.

When the chosen number of frequency components, $K$, is too small then unintended frequencies will leak and pollute the filtered data. In the next section we experiment with increasing $\mathrm{K}$ and find a sufficiently sharp filter. For subsequent analysis, our streamfunction dataset is represented in terms of spherical harmonics, where $m$ is the zonal and $n$ the total wavenumber,

$$
\Psi(\lambda, \mu, t)=\sum_{m} \sum_{n} \Psi_{m n}(t) P_{n}^{m}(\mu) e^{i m \lambda} .
$$

Here $\Psi_{-\mathfrak{m} n}(t)=\Psi_{m n}^{*}(t)$ where $*$ denotes complex conjugation, $\lambda$ is the longitude, $\mu$ is the sine of the latitude and $P_{\mathfrak{n}}^{m}(\mu)$ are Legendre functions.

\section{POPs and EOFs}

In this section we firstly obtain the leading storm track patterns associated with the complete high pass filtered dataset of Section 2. We fit a linear stochastic model to the data, of the form

$$
\frac{\mathrm{d} x}{\mathrm{dt}}=\mathbf{M x}(\mathrm{t})+\mathbf{f}(\mathrm{t}),
$$

where $\mathbf{x}$ is the column vector of spherical harmonic spectral coefficients $\Psi_{m n}$ in (2). The matrix $\mathbf{M}$ is to be determined from the data and $\mathbf{f}(\mathbf{t})$ represents 
noise. Since our data is sampled every $\Delta t=6$ hours, we estimate the stability matrix $\mathbf{M}$ through the associated finite-difference equation

$$
\mathbf{x}(\mathrm{t}+\Delta \mathrm{t})=(\mathbf{I}+\Delta \mathrm{t} \mathbf{M}) \mathbf{x}(\mathrm{t})+\Delta \mathbf{t} \mathbf{f}(\mathrm{t})
$$

where $\mathbf{I}$ is the unit matrix. The estimate of $\mathbf{M}$ that minimises the noise is then given through Gauss' theorem of least squares [3] as

$$
\mathbf{M}=\left[\left\langle\mathbf{x}(\mathrm{t}+\Delta \mathrm{t}) \mathbf{x}^{+}(\mathrm{t})\right\rangle\left\langle\mathbf{x}(\mathrm{t}) \mathbf{x}^{+}(\mathrm{t})\right\rangle^{-1}-\mathbf{I}\right](\Delta \mathrm{t})^{-1},
$$

where the + superscript denotes the Hermitian conjugate, and angular brackets denote time means. The covariance of the noise (not required here) is given in (2.4) of the paper of Frederiksen and Branstator [3]. The empirical orthogonal functions (EOFs) are the eigenvectors of $\left\langle\mathbf{x}(\mathbf{t}) \mathbf{x}^{+}(\mathbf{t})\right\rangle$ while the empirical normal modes or principal oscillation patterns (POPs) are the eigenvectors of $\mathbf{M}$. To calculate the POPs, we assume the perturbations have a time dependence $e^{-i \omega t}$. Here $\omega=\omega_{r}+i \omega_{i}$ is the complex angular frequency, $\omega_{r}$ the frequency and $\omega_{i}$ the growth rate. This then results in the system of eigenvalue-eigenvector equations

$$
-i \omega e=\mathrm{Me},
$$

where $e$ is the column vector consisting of spherical harmonic spectral coefficients of the field variables and $\mathbf{M}$ is the dynamical matrix in (5). A rhomboidal $\mathrm{R}=15$ truncation is used in which the zonal wave number $\mathrm{m}=-15, \ldots, 15$ and the total wave number $\mathrm{n}=|\mathrm{m}|,|\mathrm{m}|+1, \ldots,|\mathrm{m}|+15$ giving a total of $\mathrm{N}=496$ POPs. This corresponds to a resolution of $750 \mathrm{~km}$ by $500 \mathrm{~km}$ in longitude by latitude at $30^{\circ} \mathrm{S}$, and is adequate for resolving the leading synoptic weather modes and lower frequency modes of variability.

The POPs are sustained by the random forcing, and decay in its absence, so that the stationary time series can be reconstructed. The EOFs, eigenvectors of the covariance matrix, determine the variance of the fluctuations explained.

The leading EOF in Figure 1(a) is the maximum variance eigenvector and illustrates the familiar wave train pattern we expect to see for storm track 
Figure 1: SH 1949-1968 leading (a) EOF and (b) POP, and (c) EOF1 and (d) EOF3 for growth rates $\geqslant 0.8$ day $^{-1}$.

(a)

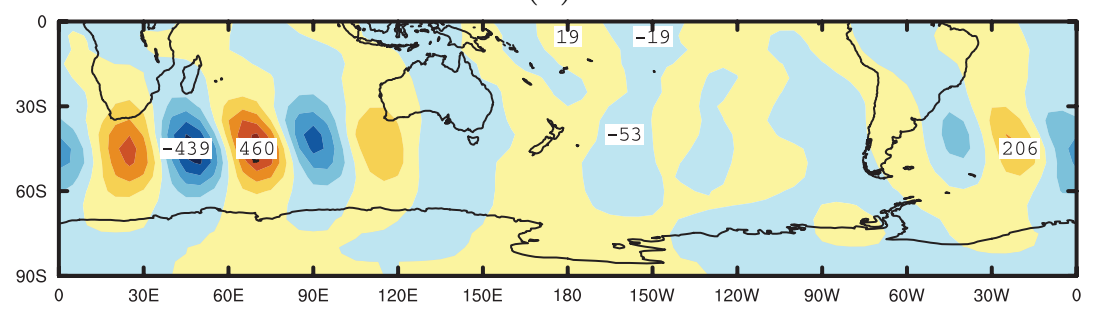

(b)

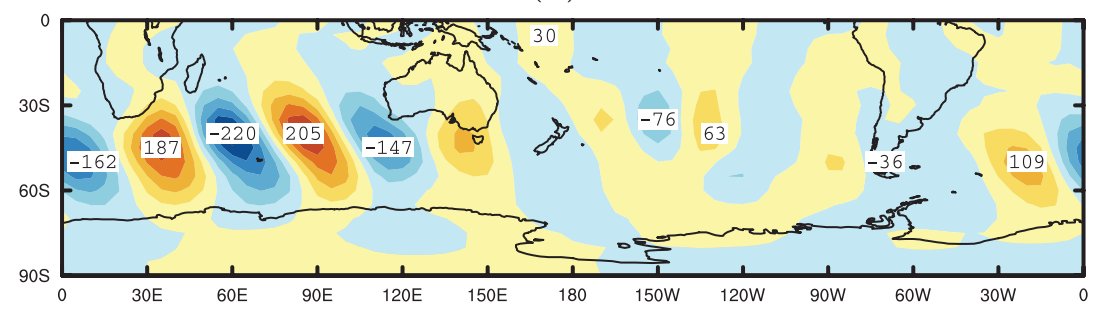

(c)

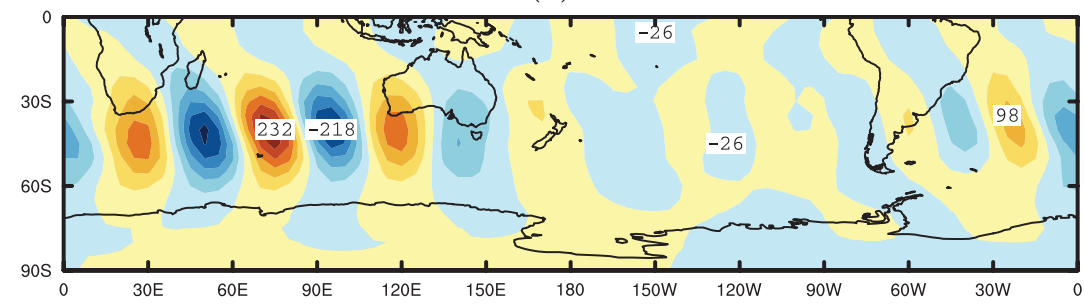

(d)

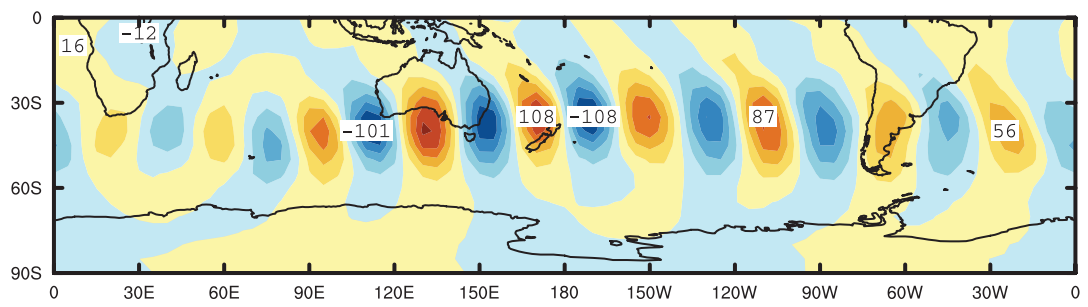


propagation upstream and over Australia. The leading POPs are those with the longest damping times. The leading POP, Figure 1(b), shows a similar wave train pattern which propagates further across Australia and has frequency $\omega_{\mathrm{r}}=0.285$ day $^{-1}$ with decay rate $-\omega_{i}=0.522$ day $^{-1}$. We test the accuracy of our filter by determining how well the frequencies are captured with varying values of $\mathrm{K}$. Our findings show that for $\mathrm{K}=40$ the filter leakage is insignificant, with the first 60 modes having periods less than four days. The POPs have the representation

$$
\Psi_{m n}(\lambda, \mu, t)=\sum_{m} \sum_{n} \psi_{m n}^{v} e^{\left(\omega_{i}^{v}-i \omega_{r}^{v}\right) t} P_{n}^{m}(\mu) e^{i m \lambda}+\text { c.c. }
$$

where the mode index $v=1, \ldots, N$, c.c. denotes the complex conjugate and a general disturbance can be represented in terms of the $\mathrm{N}$ (nondegenerate) POPs or EOFs [2].

\section{Computation of growing disturbances}

Next we determine the statistics of growing storms, and their decadal changes, for different growth rate (and decay rate) bands from the high pass filtered dataset in Section 2. We again assume a time dependence of the spectral coefficient of the form $e^{-i \omega(m, n) t}$ where $\omega=\omega_{r}(m, n)+i \omega_{i}(m, n)$, so that

$$
\Psi_{m n}(t) \equiv \psi_{m n} e^{\omega_{i}(m, n) t} e^{-i \omega_{r}(m, n) t} .
$$

Then the growth rate $\omega_{i}(m, n)$ between $t$ and $t+\Delta t$ is determined by

$$
\left\{\begin{array}{l}
\frac{\Psi_{m n}(t+\Delta t)}{\Psi_{m n}(t)}=e^{\omega_{i}(m, n) \Delta t} e^{-i \omega_{r}(m, n) \Delta t}, \\
\omega_{i}(m, n)=\frac{1}{\Delta t} \ln \left|\frac{\Psi_{m n}(t+\Delta t)}{\Psi_{m n}(t)}\right|
\end{array}\right.
$$


We can also determine the frequency

$$
\omega_{r}(m, n)=-\frac{1}{\Delta t} \arctan \left[\frac{\mathfrak{I}\left(\frac{\Psi_{\mathfrak{m} n}(t+\Delta t)}{\Psi_{m n}(t)}\right)}{\mathfrak{R}\left(\frac{\Psi_{m \mathfrak{n}}(t+\Delta t)}{\Psi_{m n}(t)}\right)}\right] .
$$

In our calculations of $\omega_{i}(m, n)$ we use a time step of $\Delta t=6$ hours. The covariances of weather systems in different growth rate bands are determined directly from observed flows allowing the separation of decaying and growing modes. The leading EOFs in each of the chosen growth rate bands are then derived. Our focus is on midlatitude winter storm tracks and for this reason we examine the meridional velocity EOFs during the early period (1949-1968) for July at $850 \mathrm{hPa}$.

Figure 1(c) and Figure 1(d) show some of the leading EOFs of the most rapidly growing storms with growth rate values greater than 0.8 day $^{-1}$. The first EOF shows a pattern very similar to Figure $1 \mathrm{a}$ with a wave train pattern across the Southern Ocean between Africa and Australia. The second EOF (not shown) also has a similar pattern, but is phase shifted. The third EOF has an extensive wave train pattern which travels across the whole hemisphere. The leading EOFs for the period (1997-2006) have very similar structures. When we compare Figure $1 \mathrm{~d}$ with that from the primitive equation instability model from Figure 3 in the paper by Frederiksen et al. [6], we find a similar wave train pattern which propagates eastward over Australia and into the South Pacific.

Next, we calculate the standard deviation of the disturbance streamfunction from each of the covariance matrices of chosen growth rate bands. Figure 2 shows differences in six hourly evolved standard deviations of streamfunction at $850 \mathrm{hPa}$ for July between the period during the AMD (1997-2006) and the earlier time period (1949-1968). By using observational reanalysis data instead of parameterised model data, we are able to composite snapshots of events with realistic values of large growth rates. Undoubtedly, as storms grow they must also decay with time, but this happens at varying rates. 
Figure 2(d) shows that during the AMD fewer storms formed rapidly in the Australian region, however not all these storms decayed rapidly (Figure 2(a)). Our results broadly agree with those from the instability model with an expected increase of the onset of storms along the polar jet during the later period of the AMD. Our fastest growing modes also show a reduction over Australia during the AMD, in particular over the southern and south-eastern regions and tracking towards New Zealand. This is consistent with the decline in number of occurrences of low pressure systems known as East Coast Lows that are responsible for bringing widespread rainfall along the east coast of Australia during winter [5].

The instability calculations of previous studies are known to underestimate the number of extremes for a 20 year July average with a maximum growth rate of 0.423 day $^{-1}$ during $1949-1968$. The advantage of our method is its ability to analyse realistic flow data to discriminate between slowly growing and fast growing modes that are more likely to result in stronger storm activity. A range of statistics about growing disturbances of a particular frequency in a given location will in turn provide us with information related to extreme weather activity.

\section{Conclusion}

We have formulated a new computational technique for calculating realistic growth rate values using observational reanalysis atmospheric data which eliminates the constraints encountered from parameterised model simulations. This technique allows a range of statistics to be calculated on growing disturbances for a given frequency at any location. The focus of this study is on changes during the period of the Australian Millennium Drought and the behaviour of storm tracks in the southern hemisphere relating to Australian rainfall. Principal component analysis methods are used to check that the filtered cyclogenesis data does indeed have frequencies less than 4 days. We 
Figure 2: Difference in six hourly evolved standard deviation of July streamfunction in $\mathrm{km}^{2} \mathrm{~s}^{-1}$ at $850 \mathrm{hPa}$ between the period during drought (1997-2006) and the earlier period (1949-1968). (a) rapidly decaying, $\omega_{i}<-0.8$ day $^{-1}$, (b) slowly decaying, $-0.8 \leqslant \omega_{i}<0.0$ day $^{-1}$, (c) slowly growing, $0.0 \leqslant \omega_{i}<$ 0.8 day $^{-1}$, and (d) rapidly growing, $\omega_{i} \geqslant 0.8$ day $^{-1}$.

(a)

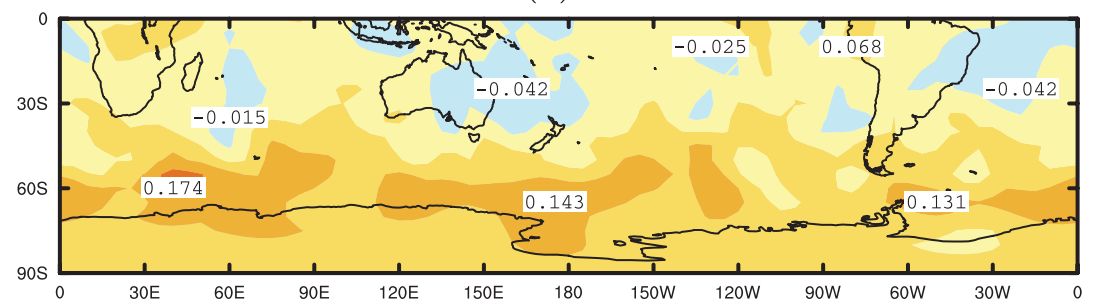

(b)

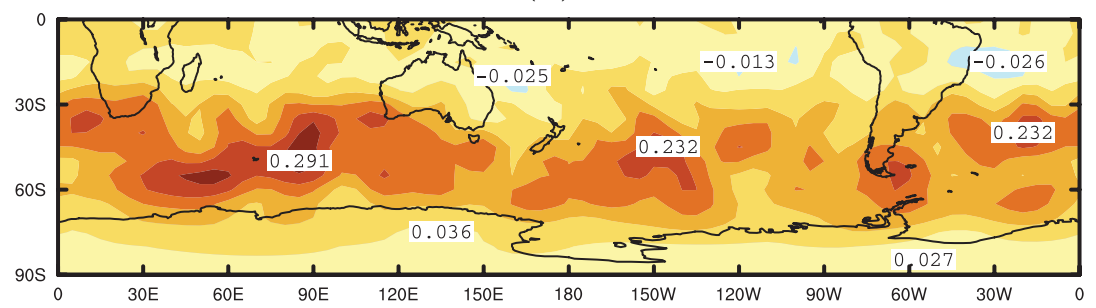

(c)

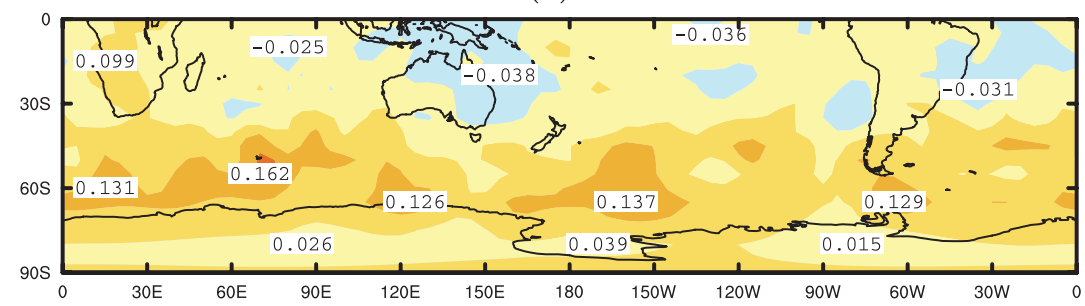

(d)

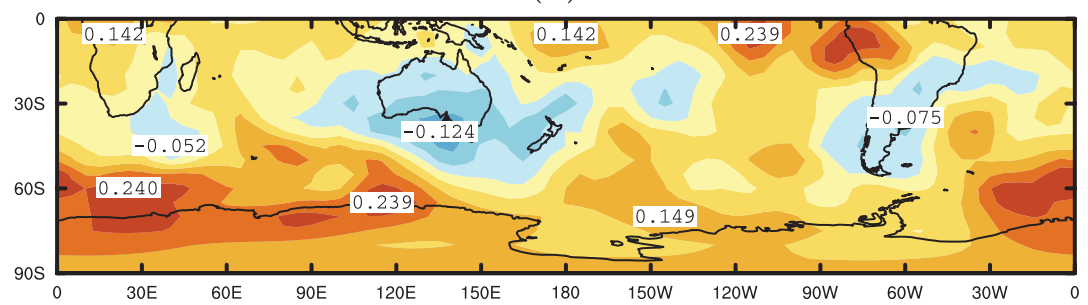


find our results to be consistent with previous modelling studies showing the weakening of the subtropical jet during winter and its apparent poleward shift, as well as the decline in number of extratropical storms including East Coast Lows.

Detailed studies of storm formation means a better understanding of the statistics of extreme weather events. Future development will include detection of smaller scale features by increasing the resolution and the number of growth rate bands. Further studies will include using different filters for atmospheric blocking and intraseasonal variability patterns. This will be extended to include covariability of rainfall with regions of high storm activity plus projections of prolonged dry periods using data from skilful global climate models.

Acknowledgements This project is supported through funding from the Australian Government's National Environmental Science Programme. Support was also provided by the Australian Research Council's Centre of Excellence for Climate System Science.

\section{References}

[1] M. Baxter and R. G. King, Measuring business cycles: Approximate band-pass filters for economic time series. The Review of Economics and Statistics 81(4):575-593, 1999. doi:10.1162/003465399558454 C140

[2] J. S. Frederiksen and R. C. Bell, North Atlantic blocking during January 1979: Linear theory, Q. J. R. Meteorol. Soc. 116:1289-1313, 1990. doi:10.1002/qj.49711649603 C143

[3] J. S. Frederiksen and G. Branstator, Seasonal variability of teleconnection patterns. J. Atmos. Sci. 63:1346-1365, 2005. doi:10.1175/JAS3405.1 C141 
[4] J. S. Frederiksen and C. S. Frederiksen, Interdecadal changes in southern hemisphere winter storm track modes. Tellus, A 59:599-617, 2007. doi:10.1111/j.1600-0870.2007.00264.x C138

[5] J. S. Frederiksen and C. S. Frederiksen, Role of dynamical modes in changing southern hemisphere climate. ANZIAM J. 52:C56-C72, 2011. doi:10.21914/anziamj.v52i0.3892 C138, C145

[6] C. S. Frederiksen, J. S. Frederiksen, J. M. Sisson and S. L. Osbrough, Trends and projections of storm formation in coupled climate models. ANZIAM J. 56:C279-C295, 2014. doi:10.21914/anziamj.v56i0.9406 C138, C144

[7] C. S. Frederiksen, J. S. Frederiksen, J. M. Sisson and S. L. Osbrough, Trends and projections of southern hemisphere baroclinicity: the role of external forcing and impact on Australian rainfall. Clim Dyn.

48:3261-3282, 2017. doi:10.1007/s00382-016-3263-8 C138

\section{Author addresses}

1. S. L. Osbrough, CSIRO, Aspendale, Victoria 3195, Australia and Monash University, Clayton, Victoria 3800, Australia.

mailto:stacey.osbrough@csiro.au orcid:0000-0001-6999-2405

2. J. S. Frederiksen, CSIRO, Aspendale, Victoria 3195, Australia and Monash University, Clayton, Victoria 3800, Australia. 